PREPARED FOR SUBMISSION TO JINST

$3^{\text {RD }}$ European Conference on Plasma Diagnostics

$6^{\text {TH }}-9^{\text {TH }}$ MAY 2019

LisBon, PORTUGAL

\title{
Bolometer developments in diagnostics for magnetic confinement fusion
}

\author{
H. Meister, ${ }^{a, 1}{ }^{1}$ M. Bernert, ${ }^{a}$ W. Biel ${ }^{b}$ M. Han ${ }^{c}$ L. C. Ingesson ${ }^{d}$ K. Mukai ${ }^{e}$ F. Penzel ${ }^{a}$ B. J. \\ Peterson $^{e}$ R. Reichle ${ }^{f}$ M. L. Reinke ${ }^{g}$ S. Schmitt ${ }^{h}$ D. Zhang $^{i}$ \\ ${ }^{a}$ Max-Planck-Institut für Plasmaphysik, Garching, Germany \\ ${ }^{b}$ Forschungszentrum Jülich GmbH, Institut für Energie- und Klimaforschung — Plasmaphysik, Jülich, \\ Germany \\ ${ }^{c}$ Michigan State University, East Lansing, Michigan, USA \\ ${ }^{d}$ Fusion for Energy, Barcelona, Spain \\ ${ }^{e}$ National Institute for Fusion Science, Toki, Japan \\ ${ }^{f}$ ITER Organization, St.-Paul-lez-Durance, France \\ ${ }^{g}$ Oak Ridge National Laboratory, Oak Ridge, Tennessee, USA \\ ${ }^{h}$ Fraunhofer-IMM, Mainz, Germany \\ ${ }^{i}$ Max-Planck-Institut für Plasmaphysik, Greifswald, Germany \\ E-mail: meister@ipp.mpg.de
}


Aвstract: The plasma radiation is an essential part of the power balance in current and future magnetic confinement fusion experiments and gives crucial insight for the challenges of power exhaust and divertor detachment as well as valuable information to understand plasma instabilities and transport effects. It is typically measured using various types of bolometers. Present day experimental devices, both the tokamak and stellarator, make use of metal resistor bolometers and infrared imaging video bolometers (IRVB), depending on the main focus of the respective measurement. The well-established sensor for absolutely calibrated measurements is the metal resistor bolometer. AXUV diodes, often used in conjunction with bolometers, are ideal for observing fast transient events in a plasma due to their very short response times, but their sensitivity varies significantly over the full radiation spectrum and degrades over their lifetime. In cases where many lines-of-sight are needed to observe radiation profiles in complex geometries IRVB offers the ability to integrate high channel counts in rather narrow installation volumes. Fibre-optic bolometers are a new development promising measurements immune to electro-magnetic interference. These diagnostic concepts are presented as well as their pros and cons.

For future devices like ITER and DEMO, R\&D efforts are required to adapt sensors and diagnostic schemes to the harsh nuclear environment. An overview will be given over the activities for sensor development and integration challenges, which may also be relevant for long pulse operation in present experiments.

KeYwords: Photon detectors for UV, visible and IR photons (vacuum), Nuclear instruments and methods for hot plasma diagnostics, bolometer, ITER, DEMO 


\section{Contents}

1 Introduction 1

2 Bolometer diagnostic principles $\quad 2$

3 Bolometer sensor types 3

3.1 Resistive bolometers 3

3.2 Infrared imaging video bolometers 5

3.3 AXUV diodes 6

3.4 Fibre optic bolometers 6

$\begin{array}{lll}3.5 & \text { Ferro-electric bolometers } & 7\end{array}$

4 Developments for current and future devices $\quad 7$

4.1 Mechanical stability of resistive bolometers at high temperatures 8

4.2 Spectral sensitivity of bolometer sensors 8

4.3 Shielding against mm-wave stray radiation 9

$\begin{array}{ll}4.4 \text { Tackling thermal drifts } & 10\end{array}$

$\begin{array}{ll}4.5 & \text { IRVB concept for ITER }\end{array}$

5 Radiation measurement concept for DEMO

6 Conclusions 12

\section{Introduction}

Plasma radiation is an essential part of the overall power balance in present and future fusion devices. In steady state conditions the power input to the plasma from fusion reactions and external heating input is balanced by the convective and conductive losses to the first wall and the power emitted through electromagnetic radiation and generated by nuclear reactions, i.e. the power due to the kinetic energy of neutrons and alpha particles from the fusion reactions. In case of non-stationarity, in particular when the plasma changes confinement states like in transitions between low and high confinement regimes or during disruptions, the temporal change of the plasma energy has to be considered, too. As part of this power balance, the plasma radiation can be used to tailor the thermal load to the wall, is an important indicator of instabilities and transport processes and can trigger disruptions or help mitigate them.

The expected heat flux densities to the target elements of the divertor in ITER can reach values up to $50 \mathrm{MW} / \mathrm{m}^{2}$ if not mitigated and for DEMO this can exceed $300 \mathrm{MW} / \mathrm{m}^{2}$. As material limits are only of the order of $10 \mathrm{MW} / \mathrm{m}^{2}$ in ITER and reduced to $5 \mathrm{MW} / \mathrm{m}^{2}$ in DEMO due to the higher neutron fluences [1]. Consequently, the power dissipation due to radiation needs to be increased 
to values of $95 \%$ or higher. This can be achieved by impurity seeding with elements like N, Ne, $\operatorname{Ar}$ or $\mathrm{Kr}[2,3]$. However, to control the radiated fraction a good signal accuracy will be required to be able to distinguish between few percent of the measured total radiation as a radiated fraction above $100 \%$ will result in the radiative collapse of the plasma. All these elements will produce different radiation distributions. In particular in the divertor region plasma simulations predict very localized radiation patterns $[4,5]$. To diagnose them a good spatial resolution will be needed.

Disruptions are an abrupt end to a plasma discharge which can happen for a variety of reasons. Common to all disruptions is that the energy of the plasma is dissipated in a very short time of the order of ms resulting in some of the strongest light sources in the world and also to very high forces on all machine parts [6,7]. Thus, the effect of disruptions need to be mitigated by dissipating the plasma energy as an evenly distributed radiation instead of allowing localized heat fluxes and forces. Accordingly, radiation measurements can help to study and improve disruption mitigation methods. However, a high temporal resolution of $1 \mathrm{~ms}$ or less is required.

Bolometers are typically used to measure the plasma radiation. A good overview of bolometer diagnostics, their original developments and applications up to 2008 have been given in [8]. This article aims at reviewing the recent developments of bolometer sensor types and diagnostic design aspects. After a brief introduction to the principles of a bolometer diagnostic in section 2 the various types of bolometer sensors will be presented in section 3, discussing measurement principles as well as advantages and drawbacks. Section 4 gives then an overview of the recent and ongoing developments relevant for present and future fusion devices. Finally, section 5 presents an outlook towards a potential concept for radiation measurement on a reactor like device as DEMO before concluding.

\section{Bolometer diagnostic principles}

A bolometer consists in general of an absorber and a thermometer (figure 1). The radiative power to be measured is deposited into the absorber and thus increases its temperature. This increase in temperature is monitored by the thermometer which translates the temperature increase into a measurement signal proportional to the absorbed radiated power. According to this measurement

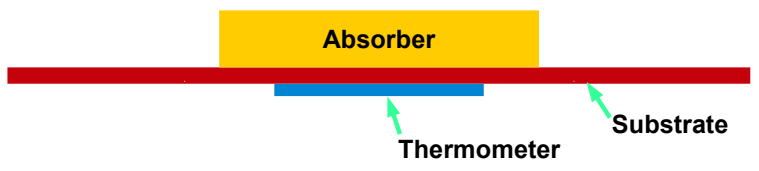

Figure 1. Bolometer principle: the radiated power is deposited in the absorber whose temperature increase is measured by a thermometer and thus generating a measurement signal. The substrate carrying absorber and thermometer has to allow efficient thermal transfer between absorber and thermometer.

principle bolometers detect the total radiated power, integrated over a wide energy spectrum, typically from the near IR up to the soft-X energy range.

The region of the plasma from which radiation is detected in each sensor is defined by the geometry of the camera. Pin-hole or collimator cameras must be used to define the desired fields of view; refractive or collimating optics would absorb part of the energy spectrum to be detected 
and thus prevent the diagnostic from actually measuring the total radiation. Detailed descriptions of how the geometry of these camera types can be calculated are given in [8-10].

A bolometer sensor measures the radiative power only integrated along a line-of-sight (LOS). By using the measurements from many LOS, the local radiation emission profile can be reconstructed. The LOS integrated signal $f_{i}$ for each LOS $i$ is given by

$$
f_{i}=\iint K_{i}(x, y) g(x, y) d x d y,
$$

with the $2 \mathrm{D}$ emission profile $g(x, y)$ and the geometry of the LOS viewing cone $K_{i}(x, y)$. Inversion of such an integral equation is often classified as an ill-posed problem, for which there is no unique solution because of the finite sampling, and a differential operator in the inverse amplifies noise present in the input data [8]. Many different inversion methods have been devised over the years to calculate $g(x, y)$ from the LOS geometry and the measured LOS integrated signals using a variety of regularization methods. The latter make use of additional knowledge about the specific arrangement at hand to restrict the number of solutions. Many publications for those exist in literature. A good overview of the problem and the major regularisation methods employed for magnetic confinement fusion is given in [8]. Without going further into details in this article, let it suffice to point out that, in order to achieve a correct interpretation of the results one has to account in the reconstruction for measurement errors of $f_{i}$ and the correct widening of the field of view. In particular for the latter, recent investigations have re-emphasized that the partial vignetting of the field of view or/and LOS passing through regions with strong gradients in the radiation emission require a thorough treatment of the LOS geometry $[10,11]$.

\section{Bolometer sensor types}

\subsection{Resistive bolometers}

Resistive bolometers detect the temperature change in the absorber by a change of resistance in a metallic resistor placed underneath the absorber (see figure 2). Two absorbers, measurement

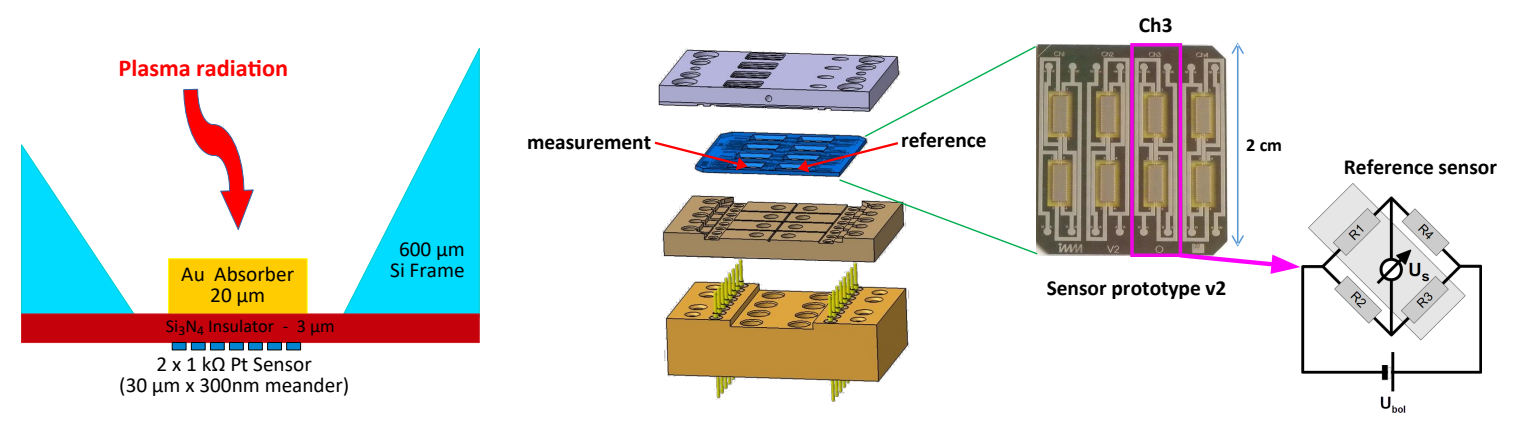

Figure 2. Principle of the resistive bolometer. Plasma radiation heats up the absorber (left). Measurement and reference absorbers are combined within one channel (middle). Electrical resistors in a meander shape underneath the absorbers are combined in a Wheatstone bridge configuration (right) yielding a sensitive signal proportional to the temperature increase of the measurement absorber due to plasma radiation.

and reference absorber, are combined within one bolometer channel while the reference absorber 
is shielded from plasma radiation. The resistors underneath both absorbers are combined in the electrical scheme of a Wheatstone bridge. This results in a very sensitive signal proportional to the incoming plasma radiation. Temperature changes affecting both, measurement and reference absorber, equally, like e.g. environmental temperature, are compensated and do not influence the signal. The first miniaturised and integrated sensor of this type has been developed in 1991 [12]. The incident time dependent plasma radiation $P_{\mathrm{rad}}(t)$ can be approximated by

$$
P_{\mathrm{rad}}(t)=\frac{C_{1} \kappa}{U_{\mathrm{bol}}} \cdot\left[\tau \frac{d U_{S}(t)}{d t}+C_{2} U_{S}(t)\right]
$$

$C_{1}$ and $C_{2}$ are constants of the electrical circuit, $U_{\mathrm{bol}}$ is the voltage driving the Wheatstone bridge, $U_{S}$ the resulting imbalance of the bridge and the measurement signal. $\tau$ and $\kappa$ are the calibration constants denoting the cooling time constant and normalised heat capacity of the sensor, respectively. These calibration constants can be determined in-situ before every plasma discharge by applying a voltage to the bridge and measuring the resulting current and its decay during the heating up of the absorber. A detailed derivation of the corresponding equations (leading ultimately to eq. 3.1) and the definitions of the constants are given in [13]. An alternative scheme to calibrate and operate resistive bolometers has recently been established having simpler and less expensive data acquisition electronics in mind [14].

The scheme of absolute in-situ calibration laid the basis for resistive bolometer sensors to advance to the state-of-the-art sensor for absolute radiation measurements in magnetic confinement fusion worldwide. However, a drawback of this sensor is that using a Wheatstone bridge configuration for measurements requires four signal wires for each channel. This results in a high amount of cabling for this diagnostic and also in its sensitivity to noise due to electro-magnetic interference (EMI). Furthermore, while the layout of this sensor with measurement and reference absorber makes it insensitive to elevated environmental temperature it does make it sensitive to any thermal gradients between the two absorbers. This will be discussed in slightly more detail in section 4.4 .

The theoretical limit of the latency for the resistive bolometers depends on the thermal transfer time between depositing the radiative power in the absorber and detecting this temperature increase in the resistors underneath. It depends on the thermal conductivity and thickness of the materials used and varies typically between $2 \mu \mathrm{s}$ and $2 \mathrm{~ms}$ [15]. In reality, resulting time resolutions depend on the sampling rate of the data acquisition electronics and the noise of the signal. Typically, the latter requires evaluating a time average of the measured bridge imbalance as the calculation of the radiated power involves a time derivative (see eq. 3.1) and thus enhances any picked up noise. This leads to typical time resolutions in current devices of the evaluated radiated power in the order of $10 \mathrm{~ms}$, which can be reduced to $1 \mathrm{~ms}$ if the signal to noise ratio is high.

The noise level of this sensor strongly depends on the time resolution employed. Noise equivalent powers of as low as $0.2 \mu \mathrm{W}$ could be measured under laboratory conditions using long integrations times [16]. Current devices like e.g. ASDEX Upgrade show noise levels between $20 \mu \mathrm{W}$ and $800 \mu \mathrm{W}$, depending on channel and the data treatment applied to filter the derivative in eq. 3.1. The main reason for the enhanced noise level compared to the ideal laboratory environment is EMI pick-up in the many signal cables which are difficult to shield absolutely in fusion devices. 


\subsection{Infrared imaging video bolometers}

In the case of infrared imaging video bolometers (IRVB) a thin metallic foil is heated by plasma radiation and the temperature distribution on the foil is monitored by an IR camera. The principle of an IRVB pinhole camera is sketched in figure 3 and presented in detail in [17, 18]. To determine

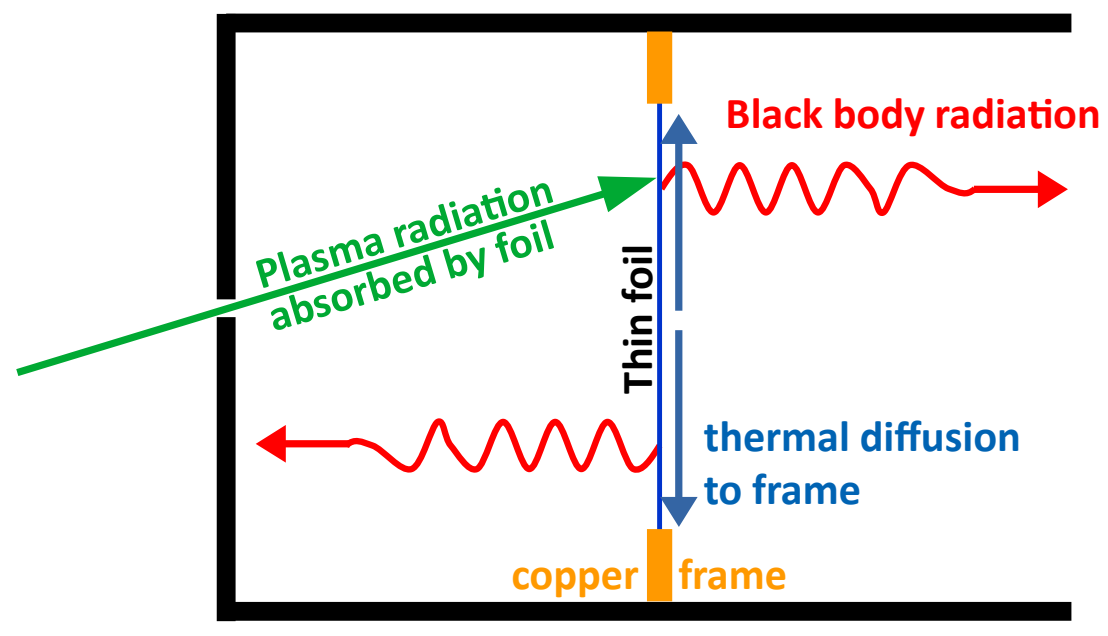

Figure 3. Sketch of an IRVB pinhole camera indicating the sources and sinks determining the temperature distribution on the thin metallic foil (typically $7 \mathrm{~cm} \times 9 \mathrm{~cm}, 2.5 \mu \mathrm{m}$ thick).

the incident radiative power, $P_{\text {rad }}$, from the measured foil temperature distribution $T$, one has to solve the two-dimensional heat diffusion equation for the foil:

$$
\frac{\partial^{2} T}{\partial x^{2}}+\frac{\partial^{2} T}{\partial y^{2}}=\frac{P_{\mathrm{rad}}}{k t_{f} l^{2}}+\frac{1}{\kappa} \frac{\partial T}{\partial t}+\frac{\epsilon \sigma_{S-B}\left(T^{4}-T_{0}^{4}\right)}{k t_{f}}
$$

Accordingly, the 2D-distribution of the temperature on the foil is determined by the incoming plasma radiation, the thermal diffusivity towards the supporting copper frame and the black body radiation of the blackened foil. $l$ denotes the size of a bolometer pixel as imaged by the pixels of the IR camera. $\epsilon$ is the emissivity of the foil, which is blackened and thus kept as close to 1 as possible. The foil thermal conductivity $k$, its thickness $t_{f}$ and its thermal diffusivity $\kappa$ are the calibration constants. They depend on the material of the foil and show a spatial variation due to tolerances of the manufacturing process. Therefore, the IRVB requires a demanding 2D calibration procedure.

The advantage of the IRVB is that it can provide a large number of LOS within a relatively small space envelope. The number is determined by the number of pixels of the IR camera onto which the image of the foil is mapped by the observing optics. Thus, using sensitive state-of-the-art IR cameras with typically $1024 \times 1280$ pixels allow a binning of $40 \times 40$ camera pixels into one bolometer pixel resulting in about 768 LOS within one camera. However, IRVB views are typically oriented toroidally which makes it difficult to conclude on the achievable spatial resolution from the amount of LOS only; the quality of the data and the inversion method applied have a strong impact on the kind of radiation patterns which can be resolved. As IR cameras operate usually with frame rates in the order of $100 \mathrm{~Hz}$ for the full frame, time resolutions of $10 \mathrm{~ms}$ can be achieved in an optimal case. 
This type of diagnostic has first been established in 2000 on LHD [17]. Meanwhile, this diagnostic is operational on LHD, K-Star, Aditya-U and GAMMA10/PDX. It has also been operated at JT60-U, Alcator C-Mod and SST-1, is installed on MAST-U and conceptual design studies are in progress for Heliotron J, W7-X and ASDEX Upgrade.

\subsection{AXUV diodes}

AXUV diodes measure photons by a photo current generated in a p-n-junction with very high quantum efficiency [19]. They are often used in conjunction with bolometers to detect radiation since the early 1990s. The resulting photo-current should, in principle, be proportional to the incident radiative power. Unlike resistive bolometers, AXUV diodes are neither sensitive to neutral particles with energies below $200 \mathrm{eV}$ nor pressure changes. But in principle electrons, ions, neutrons and higher energetic neutral particles may create electron-hole pairs in the diodes and thereby causing a parasitic signal. Nonetheless, due to their easy way of use, low investment costs, the low noise and the high time resolution with sampling rates of up to $2 \mathrm{MHz}$ they provide a unique opportunity to measure fast radiation events. These advantages explain their use on most fusion experiments world-wide.

However, their sensitivity is constant only for photon energies above $\approx 200 \mathrm{eV}$. Below this value, they show a strong dependency on the photon energy which makes the interpretation of the measurement signal as total plasma radiation difficult if not impossible. Furthermore, it has been observed that the diodes degrade in their efficiency during the exposure to an active experiment [20]. The impact on measurements of radiation emission profiles could clearly be observed e.g. in measurements on Alcator C-Mod, where it was demonstrated that radiation emission profiles deduced from measurements of AXUV diodes are consistent with those from resistive bolometers in the plasma center but deviate strongly in the outer half [21]. This is due to the temperature distribution in a fusion device. At the high core temperatures $\left(T_{e} \geq 500 \mathrm{eV}\right)$, the major part of the radiation results in photon energies for which diodes have an optimal sensitivity. For the radiation at the edge $\left(T_{e} \leq 500 \mathrm{eV}\right)$, where the dominant radiation power is emitted in the VUV and VIS range, diodes have a reduced sensitivity and strong dependence on the photon energy and usually measure too low signals.

\subsection{Fibre optic bolometers}

Fibre optic bolometers are a very new development which started off about three years ago with first prototypes and measurements in the lab [22] and recent improvements to enhance the achievable signal-to-noise ratio (SNR) [23]. In this case the miniature thermometer is also the absorber (figure 4): a small silicon disc of about $300 \mu \mathrm{m}$ diameter and $75 \mu \mathrm{m}$ thickness is attached to the end of a single mode optical fibre. This results in a Fabry-Perot interferometer (FPI) attached directly to the cleaved end of the optical fibre which can be interrogated by a NIR laser. The silicon absorbs the incident radiation and converts it into temperature variation which alters the optical path length of the silicon FPI and thus shifts the fringes of its reflection spectrum. The power density of the incident radiation is deduced by interrogating the fringe positions of the FPI. Introducing a Au coating on top of the silicon disc and a high-reflective dielectric coating on the bottom and using a small piece of a graded index multi-mode fibre (GI-MMF) as collimator for the laser light increased 
the sensitivity of this sensor to a noise-equivalent power density of $0.27 \mathrm{~W} / \mathrm{m}^{2}$, which is comparable to resistive bolometers.

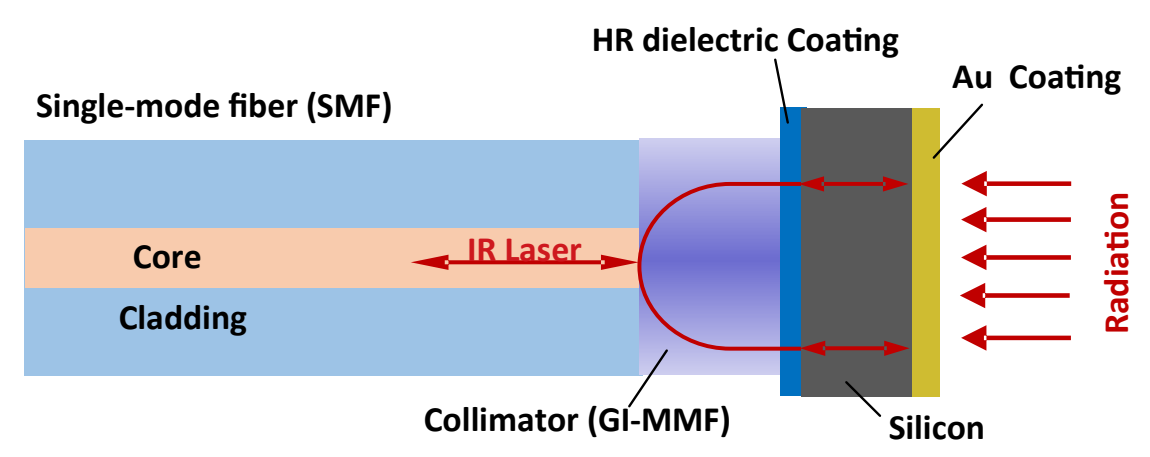

Figure 4. Principle of a fibre-optic bolometer sensor.

As the temperature change in the absorber is detected from line shifts of interference fringes and not from the measured intensity, this type of sensor is immune to the fibre browning and any EMI. Response times vary between $150 \mathrm{~ms}$ and $400 \mathrm{~ms}$, depending on the surface-to-volume ratio of the silicon pillar. This makes them relatively slow, but due to the immunity to EMI, their small size and compatibility to high operating temperatures, they are expected to support well physics studies in near-term devices.

\subsection{Ferro-electric bolometers}

Capacitive bolometers are based on a thin-film ferro-electric capacitor that exhibits large capacitance changes as a function of temperature [24]. The capacitor can be placed in a capacitive bridge in a similar fashion as the resistive bolometers but also it can be constructed as a LC resonance circuit, which makes it possible to measure a number of channels (with different resonance frequencies) with just two wires. The ferroelectric film has the promise of being relatively radiation hard and might be well suited for a burning plasma environment. However, since initial feasibility studies they were not further developed. Thus, despite the potential of ferro-electric bolometers, significant development efforts still need to be made in order to overcome the technological problems such as the unexpected difficulties in etching structures adequate for a bolometer or the adequate electrical contacts. No working prototype was installed on any fusion device so far.

\section{Developments for current and future devices}

Future fusion devices, in particular ITER, will require bolometer sensors to operate under more stringent requirements than in presently operating devices. The most obvious of these requirements is the operation under high nuclear and thermal loads. But also stray radiation from external RF heating combined with long discharge durations results in higher thermal loads and may induce a signal in the bolometer sensor which is comparable or even larger than the one from plasma radiation. The enhanced thermal loads lead to thermal gradients on support structures which result in thermal drifts of the bolometer signal if not mitigated. Furthermore, the bolometer diagnostic will have to measure the total plasma radiation reliably in a wide range of plasma scenarii under varying spectral 
distributions of the radiation, e.g. due to radiative cooling. Also, sensors have to be sensitive to high energy photons as bremsstrahlung in high temperature plasmas like in ITER is expected to show a broad spectrum up to several tens of $\mathrm{keV}$. These requirements already start playing a more and more significant role even in presently operating long-pulse devices. Accordingly, bolometer sensors have to be developed which can cope with these demands. The following sections will give a brief overview over recent development efforts focused on resistive bolometers and IRVB to meet these requirements.

\subsection{Mechanical stability of resistive bolometers at high temperatures}

The first resistive bolometer sensors developed in view of ITER used Pt absorbers on SiN membranes as these materials have low neutron cross sections and thus are resistant against the expected nuclear loads. However, albeit prototypes showed a promising sensitivity [25], thermal cycling up to temperatures of $450 \mathrm{C}^{\circ}$ leads to breaking membranes. Finite-element analysis revealed that intrinsic stresses from the manufacturing process paired with different thermal expansion coefficients for absorber and membrane leads to high localised stresses and breaking during the fatigue cycle under thermal cycling [26]. Thus, new material combinations for the sensor are being tested.

The high temperature bolometer sensors on JET used Au absorbers on a Mica foil. To overcome the variations of the material properties of the natural material Mica, a similar approach but using up to $25 \mu \mathrm{m}$ thin $\mathrm{ZrO}_{2}$ foils as self-supporting substrate is being tried as one alternative for ITER. The first set of prototypes from the ongoing development programme is being expected available for testing by the end of 2019 .

Another approach tested is to support the absorbers using flexure hinges [27], similar as for acceleration sensors. Prototypes were manufactured and successfully thermally cycled up to $450 \mathrm{C}^{\circ}$ without failures. Measurements of the deflection of the absorbers out of plane at room temperature are very well in agreement with results from FE modeling, demonstrating the good stress absorption by the flexure hinges. However, the hinges also provide a longer thermal path from the sensor to the heat sink which leads to increased values of the calibration constants $\tau$ and $\kappa$. Accordingly, the application of this variant as bolometer sensor requires further optimization.

A third approach is based on the developments for the first ITER prototypes with Pt absorbers but uses $\mathrm{Au}$ absorbers instead. The reason is that $\mathrm{Au}$ is more ductile and should thus induce lower stresses into the membrane while keeping the advantages of relatively easy handling of the sensors in the Si-frame as compared to the thin self-supporting substrates. These attempts have meanwhile been successful and produced bolometer sensors with $20 \mu \mathrm{m}$ thick absorbers to provide a sufficient absorption of high energy photons. They were thermally cycled up to $400 \mathrm{C}^{\circ}$. A good yield of 95 $\%$ of channels that withstand the thermal cycling out of the 128 tested was obtained. The failure rate saturated within the first five thermal cycles and did not change for an additional five thermal cycles. This makes this approach a very promising candidate for use in ITER. However, as with the other variants, prototypes will have to be irradiation tested before finally concluding on their expected lifetime in ITER.

\subsection{Spectral sensitivity of bolometer sensors}

In an ideal case a bolometer sensor has a spectral sensitivity of 1 over the whole spectral range. For first sensor types based on Au absorbers on a Kapton foil with an additional black Ni layer 
the efficiency was measured and confirmed to be close to unity for wavelengths between $1 \mu \mathrm{m}$ and $1 \mathrm{~nm}(1.2 \mathrm{eV}$ and $1.2 \mathrm{keV}$, respectively) [28]. Similar measurements were performed for a sensor based on Pt absorbers on SiN membranes with two different absorber thicknesses [16]. In this case the efficiency was also close to unity in the energy range between $20 \mathrm{eV}$ and $10 \mathrm{keV}$ ( $62 \mathrm{~nm}$ and $0.12 \mathrm{~nm}$, respectively). For higher energies the efficiency drops according to the reduced photon absorption coefficient and for lower energies the reflections on the pure Pt surface play an important role. As these are the energy ranges most important for plasma scenarii with a high fraction of line radiation and LOS observing colder plasma regions $\left(T_{e} \lesssim 50 \mathrm{eV}\right)$, they are of particular interest, as confirmed e.g. by measurements performed on the linear plasma device Magnum-PSI using a blackened Au absorber, which demonstrated clearly the beneficial effect of a blackened absorber [29]. Similar measurements have also been performed on TCV indicating the improvement, too [30].

Accordingly, efforts have been undertaken to develop a blackening process for the current generation of resistive bolometer sensors. Sputtered carbon coating turned out to be well compatible with the manufacturing processes and resulted in acceptable absorption coefficients above $80 \%$ for the VIS and VUV (between 400-100 nm, respectively 3.1-12 eV). Samples with this coating have been installed during the recently completed operational campaign OP1.2b in Wendelstein 7-X and showed no visible degradation so far (see figure 5), demonstrating the durability of the chosen coating. Measurements to characterise the reflectivity of the coating are ongoing.
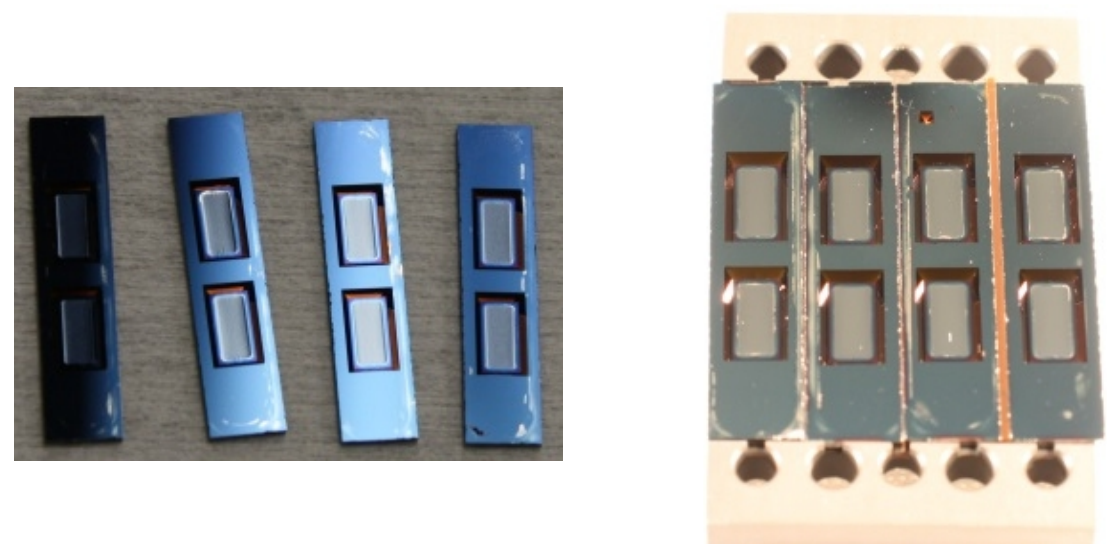

Figure 5. Photos of C-coated resistive bolometer sensors before (left) and after (right) operation in W7-X.

Blackening of the foil is also an important aspect for IRVB. In this case a vacuum evaporated carbon coating has been developed which significantly enhanced reproducibility and uniformity of the calibration process so that uncertainties less than about $10 \%$ could be achieved [31, 32].

\subsection{Shielding against $\mathrm{mm}$-wave stray radiation}

In case fusion plasmas are heated by external radio-frequency (RF) heating and the external RF power is not fully absorbed, this part is reflected multiple times by the in-vessel components and its power might get deposited even in remote places inside the vacuum vessel. In particular for longpulse experiments this can result in significant thermal loads on components and sensors if they are not shielded properly [33]. Already during the construction phase of W7-X this has been considered 
for bolometers [34] and mitigation methods proposed. But also for experiments with shorter pulse lengths mm-wave stray radiation can reach levels on bolometer sensors which are comparable to the radiation signal, as experience from EAST demonstrates [35]. Thus, also on W7-X and ASDEX Upgrade counter measures like microwave reflecting grids have been implemented in the pinhole or directly in front of the sensors. Additionally, microwave absorbing coatings like $\mathrm{Al}_{2} \mathrm{O}_{3} / \mathrm{TiO}_{2}$ have been applied to the inner side of bolometer cameras. This resulted in damping factors in the order of about 300 yielding reliable measurements.

However, in the case of ITER the estimated levels of stray radiation from electron cyclotron resonance heating might reach levels significantly higher than the expected signal levels from plasma radiation, thus requiring attenuation factors up to $10^{7}$. In a dedicated campaign prototypes of collimator cameras have been developed featuring a microwave reflecting grid and inner surfaces coated with the microwave absorbing ceramic $\mathrm{Al}_{2} \mathrm{O}_{3} / \mathrm{TiO}_{2}$. Measurements in the lab and in the test facility MISTRAL [36] demonstrated that the required attenuation factor could indeed be achieved over a broad frequency range [37]. The methods developed provide a good basis to tailor the needs of attenuating mm-wave stray radiation and ensure a design of the bolometer diagnostic which can cope with various levels of stray power.

\subsection{Tackling thermal drifts}

Bolometer sensors have shown for many years that they are sensitive to thermal drifts induced by thermal loads of various origins onto the sensor support structure. The reason for this is twofold. Firstly, the calibration constants depend on temperature as the underlying material parameters like thermal conductivity or specific heat capacity depend on temperature. This effect can be compensated by the additional measurement of the current through the Wheatstone bridge [38, 39]. The latter provides a measurement proportional to the temperature of the sensor and can be used to interpolate the correct value of the calibration parameters from previous calibration runs in the lab.

Secondly, thermal loads can lead to temperature gradients between measurement and reference absorber, thus giving rise to a bridge imbalance which cannot be distinguished from one induced by plasma radiation only. As estimated signal levels in ITER correspond to temperature increases of the measurement absorber by $0.1-0.7 \mathrm{~K}$ only and noise levels to $0.05 \mathrm{~K}$ a sensitive reaction to these gradients is expected. A detailed investigation of the impact of thermal gradients between measurement and reference absorber is presented in [40]. The need for compensation methods and maximization of sensor sensitivity as well as minimizing the intrinsic bridge imbalance (e.g. by re-designing the track layout on the sensor to be better balanced) is concluded. This is part of ongoing developments.

\subsection{IRVB concept for ITER}

The reference bolometer sensor for ITER is the resistive bolometer [41]. However, it is seen as beneficial to complement the diagnostic system at ITER with a second sensor type to reduce the risk of single mode failures by using a different technology. Also, cross comparisons between different sensor types will improve the interpretation and understanding of the measured radiation. The IRVB is seen as a potential candidate and investigations how it could be implemented in ITER are ongoing. The currently proposed concept foresees two cameras mounted in an equatorial port 
plug with a $36 \mu \mathrm{m}$ thick Pt foil and a field of view of $20 \times 15$ pixels for the plasma core and a $6 \mu \mathrm{m}$ thick Pt foil and a field of view of $24 \times 32$ pixels for the divertor region. The thick foil is needed for the core to capture the high energy photons from the plasma centre. The thinner foil for the divertor region makes use of the lower photon energies to increase sensitivity while still capturing all photons. For both cameras state-of-the-art IR cameras shall be used, typically with $1024 \times 1280$ pixels and a full frame rate of $105 \mathrm{~Hz}$. Based on simulations of the plasma radiation for plasma core (using the SANCO code) and edge (from SOLPS modelling) the expected SNR of the proposed systems could be estimated using eq. 1 in [42]. With a SNR of about 65 for the core and 37 for the divertor region the proposed systems are seen as well able to measure the plasma radiation.

To tackle the challenging calibration of the IRVB system an improved approach has been presented recently [32]. It is based on a removable unit which can be attached to the IRVB viewing system and uses a visible laser of known power to illuminate individual pixels of the detection foil. A mirror mounted on a biaxial goniometer scans the laser over the whole surface of the foil. Its resulting temperature distribution is detected by the IR camera and thus the calibration constants are measured. Combined with the improved carbon coatings (see section 4.2) calibration errors could be suppressed to less than about $10 \%$. Furthermore, the application of this system during a deuterium campaign on LHD demonstrated a good resilience against neutrons and plasma radiation from both, plasma discharges and glow discharges [32].

Open issues in view of the application in ITER are the space availability to actually integrate such systems in the fully occupied ports and the need of refractive optics to image the foil onto the IR camera without exposing it to directly streaming neutrons. Finally, mitigations for the impact of the high nuclear heating of the foil are still to be developed and tested.

\section{Radiation measurement concept for DEMO}

The plasma control system on DEMO should provide very reliable control mechanisms to prevent disruptions and overloading of plasma facing components. Accordingly, the plasma state on DEMO is foreseen to be determined by a sufficient number of measurements together with real-time plasma simulation, so-called advanced control models [43, 44]. The advanced control models shall capture most of the expected evolutions of the plasma state and allow for early reaction of the control system using slow and/or weak actuators. Unexpected events (e.g. impurity ingress, hardware failure, ... ) cannot be predicted by models. In such cases an early reaction can only be based on measurements. Diagnostics will also have to comply to the high neutron fluences in DEMO and the required high reliability and long life-time of all components. Thus, for each measurement task ideally two or more systems should be available. However, the high nuclear loads and the need for a high tritium breeding ratio will allow only for much fewer systems and views as compared to current devices or ITER. Accordingly, the final goal will be to establish a pre-defined scenario which can be handled with only few signals and slow and weak actuators. A set of suitable diagnostics was proposed in [44].

One of the potential controls to be implemented in DEMO is to keep the plasma in the high confinement regime, i.e. one needs to control the power flow over the separatrix such that it is kept above the L-H power threshold, but only with little margin to avoid an overloading of plasma facing components. For this, a control signal proportional to the plasma core radiation is needed. 
Candidates for suitable sensors are under investigation. In case one would implement a sensor based on current resistive bolometer sensors and consider all the requirements on diagnostics in DEMO mentioned above, the sensors would have to be retracted behind the port plug flanges to keep them in an area with nuclear loads comparable to those bolometer sensors in ITER will have to survive. This would give the additional advantage of having them accessible in case of failures and be able to exchange them. The corresponding LOS have been presented recently [45]. They result in very narrow viewing cones, which have to pass through ducts of $8 \mathrm{~m}$ length. Despite the high radiative power from the DEMO plasma estimations result in power on the sensor area of up to $30 \mu \mathrm{W}$. This will result in a very low SNR. However, the good news is that a measurement signal proportional to the plasma core radiation can be provided, even if very noisy.

\section{Conclusions}

Radiation measurements using bolometers play an important role in understanding and controlling magnetically confined plasmas. Resistive bolometers are the well-established sensor type for absolute radiation measurements and actively being developed for use in future devices. IRVB are a promising alternative avoiding electromagnetic interferences but require further development of the in-situ calibration for the absolute measurement of the radiative power. AXUV diodes and fibre optic bolometers complement measurements well on current devices but are not suited for enhanced nuclear loads as expected for ITER and DEMO.

Developments of sensors and components of resistive bolometers and IRVB for ITER are well under way. However, still a number of issues need to be solved, like the validation of the compatibility of sensors and components for the nuclear environment and the use in long plasma operations. Furthermore, sensors and data evaluation techniques need to be optimized to cope with the varying radiation spectra and thermal loads. In view of DEMO sensors will have to be placed in remote locations to assure sufficiently benign nuclear loads and accessibility during operation. Also, control mechanisms compatible to few and noisy signals have to be developed. However, based on current sensor technology assessments indicate that a control signal proportional to the core plasma radiation can be provided, even if it means operation close to a SNR of 1 .

\section{Acknowledgments}

The views expressed in this article are the sole responsibility of the authors and do not necessarily reflect the views of the Fusion for Energy and the ITER Organization. Neither Fusion for Energy nor any person acting on behalf of Fusion for Energy is responsible for the use, which might be made of the information in this publication.

\section{References}

[1] M. Wischmeier. High density operation for reactor-relevant power exhaust. Journal of Nuclear Materials, 463:22 - 29, 2015.

[2] R. Reichle, D. V. Bartlett, D. J. Campbell, H. Chen, J. C. Fuchs, R. M. Giannella, N. A. C Gottardi, N. Hawkes, L. D. Horton, H. J. Jäckel, L. Lauro-Taroni, A. C. Maas, R. D. Monk, M. G. O’Mullane, 
L. Porte, R. van der Linden, M. von Hellermann, and J. Wesson. Radiation in JET's mark I divertor. In B. E. Keen, P. E. Stott, and J. Winter, editors, Proceedings of the 22nd EPS conference on Controlled Fusion and Plasma Physics, Bournemouth, 3.-7. July 1995, volume 19C, pages 85-88, 1995.

[3] M. Bernert, M. Wischmeier, A. Huber, F. Reimold, B. Lipschultz, C. Lowry, S. Brezinsek, R. Dux, T. Eich, A. Kallenbach, A. Lebschy, C. Maggi, R. McDermott, T. Pütterich, and S. Wiesen. Power exhaust by SOL and pedestal radiation at ASDEX Upgrade and JET. Nuclear Materials and Energy, 12:111-118, 2017.

[4] F. Reimold, M. Wischmeier, M. Bernert, S. Potzel, D. Coster, X. Bonnin, D. Reiter, G. Meisl, A. Kallenbach, L. Aho-Mantila, and U. Stroth. Experimental studies and modeling of complete H-mode divertor detachment in ASDEX Upgrade. Journal of Nuclear Materials, 463:128 - 134, 2015.

[5] R. Pitts, X. Bonnin, F. Escourbiac, H. Frerichs, J. P. Gunn, T. Hirai, A. S. Kukushkin, E. Kaveeva, M. A. Miller, D. Moulton, V. Rozhansky, I. Senichenkov, E. Sytova, O. Schmitz, P. C. Stangeby, G. De Temmermann, I. Veselova, and S. Wiesen. Physics basis for the ITER tungsten divertor. submitted to Nuclear Materials and Energy, 2019.

[6] T. C. Hender, J. C. Wesley, J. Bialek, A. Bondeson, A. H. Boozer, R. J. Buttery, A. Garofalo, T. P. Goodman, R. S. Granetz, Y. Gribov, O. Gruber, M. Gryaznevich, G. Giruzzi, S. Günter, N. Hayashi, P. Helander, C. C. Hegna, D. F. Howell, D. A. Humphreys, G. T. A. Huysmans, A. W. Hyatt, A. Isayama, S. C. Jardin, Y. Kawano, A. Kellman, C. Kessel, H. R. Koslowski, R. J. La Haye, E. Lazzaro, Y. Q. Liu, V. Lukash, J. Manickam, S. Medvedev, V. Mertens, S. V. Mirnov, Y. Nakamura, G. Navratil, M. Okabayashi, T. Ozeki, R. Paccagnella, G. Pautasso, F. Porcelli, V. D. Pustovitov, V. Riccardo, M. Sato, O. Sauter, M. J. Schaffer, M. Shimada, P. Sonato, E. J. Strait, M. Sugihara, M. Takechi, A. D. Turnbull, E. Westerhof, D. G. Whyte, R. Yoshino, H. Zohm, Disruption the ITPA MHD, and Magnet Group. Chapter 3: MHD stability, operational limits and disruptions. Nuclear Fusion, 47(6):S128-S202, June 2007.

[7] G. Pautasso, M. Bernert, M. Dibon, B. Duval, R. Dux, E. Fable, J. C. Fuchs, G. D. Conway, L. Giannone, A. Gude, A. Herrmann, M. Hoelzl, P. J. McCarthy, A. Mlynek, M. Maraschek, E. Nardon, G. Papp, S. Potzel, C. Rapson, B. Sieglin, W. Suttrop, W. Treutterer, and ASDEX Upgrade Team. Disruption mitigation by injection of small quantities of noble gas in ASDEX upgrade. Plasma Physics and Controlled Fusion, 59(1):014046, Nov. 2016.

[8] L. C. Ingesson, B. Alper, B. J. Peterson, and J.-C. Vallet. Tomography diagnostics: Bolometry and soft-x-ray detection. In A. E. Costley and D. W. Johnson, editors, Special issue on plasma diagnostics for magnetic fusion research, volume 53, chapter 7. Fusion Science and Technology, 2008.

[9] H. Meister and S. Kalvin. Optimisation of design parameters for collimators and pin-holes of bolometer cameras. Fusion Engineering and Design, 89(12):3039-3045, 2014.

[10] M. Carr, A. Meakins, M. Bernert, P. David, C. Giroud, J. Harrison, S. Henderson, B. Lipschultz, F. Reimold, EUROfusion MST1 Team, and ASDEX Upgrade Team. Description of complex viewing geometries of fusion tomography diagnostics by ray-tracing. Review of Scientific Instruments, 89(083506), 2018.

[11] D. Vezinet, V. Igochine, M. Weiland, Q. Yu, A. Gude, D. Meshcheriakov, M. Sertoli, the ASDEX Upgrade Team, and the EUROfusion MST1 Team. Non-monotonic growth rates of sawtooth precursors evidenced with a new method on ASDEX Upgrade. Nuclear Fusion, 56(8):086001, 2016.

[12] K. F. Mast, J. C. Vallet, C. Andelfinger, P. Betzler, H. Kraus, and G. Schramm. A low noise highly 
integrated bolometer array for absolute measurement of VUV and soft-X radiation. Review of Scientific Instruments, 62(3):744-750, 1991.

[13] L. Giannone, K. F. Mast, M. Schubert, NBI Team, ECRH Team, and W7-AS Team. Derivation of bolometer equations relevant to operation in fusion experiments. Review of Scientific Instruments, 73(9):3205-3214, 2002.

[14] J. Lovell, A. Field, G. Naylor, and R. Sharples. An FPGA-based bolometer for the Mast-U super-X divertor. Proceedings of Science, 240, 2016.

[15] H. Meister, H. Eixenberger, C. Gliss, A. Kampke, M. Kannamüller, J. Koll, F. Penzel, K. Kotrocz, A. Pataki, J. Szücs, G. Veres, T. Fekete, L. Tatar, P. Detemple, K. Mpoukouvalas, and S. Schmitt. Overview of the R\&D activities for the ITER bolometer diagnostic. Proceedings of Science, 240, 2016. DOI: https://doi.org/10.22323/1.240.0079.

[16] H. Meister, M. Willmeroth, D. Zhang, A. Gottwald, M. Krumrey, and F. Scholze. Broad-band efficiency calibration of ITER bolometer prototypes using Pt absorbers on SiN membranes. Review of Scientific Instruments, 84(12), DEC 2013.

[17] B. J. Peterson. Infrared imaging video bolometer. Review of Scientific Instruments, 71(10):3696-3701, 2000.

[18] B. J. Peterson, A. Yu. Kostrioukov, N. Ashikawa, M. Osakabe, and S. Sudo. Calibration and sensitivity of the infrared imaging video bolometer. Review of Scientific Instruments, 74(3):2040-2043, 2003.

[19] R. Korde and L.R. Canfield. Silicon photodiodes with stable, near-theoretical quantum efficiency in the soft-X-ray region. In Benattar, R., editor, X-ray instrumentation in medicine and biology, plasma physics, astrophysics, and synchrotron radiation, volume 1140 of Proceedings of the Society of Photo-Optical Instrumentation Engineers (SPIE), pages 126-132, 1989.

[20] M. Bernert, T. Eich, A. Burckhart, J. C. Fuchs, L. Giannone, A. Kallenbach, R. M. McDermott, and B. Sieglin. Application of AXUV diode detectors at ASDEX upgrade. Review of Scientific Instruments, 85(3):033503, 2014.

[21] M. L. Reinke and I. H. Hutchinson. Two dimensional radiated power diagnostics on alcator c-mod. Review of Scientific Instruments, 79(10):10F306, 2008.

[22] M. L. Reinke, M. Han, G. Liu, G. G. van Eden, R. Evenblij, M. Haverdings, and B. C. Stratton. Development of plasma bolometers using fiber-optic temperature sensors. Review of Scientific Instruments, 87(11):11E708, 2016.

[23] Q. Sheng, G. Liu, M. L. Reinke, and M. Han. A fiber-optic bolometer based on a high-finesse silicon fabry-pérot interferometer. Review of Scientific Instruments, 89(6):065002, 2018.

[24] M. Di Maio, R. Reichle, and R. Giannella. Design of a ferroelectric bolometer. In Proc. 17th IEEE 0 NPSS Symp. Fusion Engineering, San Diego, October 6-10, 1997, volume 2, page 775. IEEE, 1998.

[25] L. Giannone, D. Queen, F. Hellman, and J. C. Fuchs. Prototype of a radiation hard resistive bolometer for ITER. Plasma Physics and Controlled Fusion, 47:2123-2143, 2005.

[26] H. Meister, M. Kannamüller, J. Koll, A. Pathak, F. Penzel, T. Trautmann, P. Detemple, S. Schmitt, and H. Langer. Reliability issues for a bolometer detector for ITER at high operating temperatures. Review of Scientific Instruments, 83:10D724, 2012.

[27] H. Meister, H. Langer, and S. Schmitt. An alternative geometry for bolometer sensors for use at high operating temperatures. Fusion Engineering and Design, pages 579-586, 2016. 
[28] P. T. Lang and K. F. Mast. Photoresponse of a miniaturized ultrabroad-band low-noise metal-film bolometer detector array. J. Optics, 27(1):25-29, 1996.

[29] G.G. van Eden, M.L. Reinke, S. Brons, G. van der Bijl, B. Krijger, R. Lavrijsen, S.P. Huber, R. Perillo, M.C.M. van de Sanden, and T.W. Morgan. Plasma radiation studies in magnum-PSI using resistive bolometry. Nuclear Fusion, 58(10):106006, jul 2018.

[30] U. A. Sheikh, B. P. Duval, B. Labit, and F. Nespoli. A novel carbon coating technique for foil bolometers. Review of Scientific Instruments, 87(11):11D431, 2016.

[31] K. Mukai, B. J. Peterson, S. Takayama, and R. Sano. In situ calibration of the foil detector for an infrared imaging video bolometer using a carbon evaporation technique. Review of Scientific Instruments, 87(11):11E124, 2016.

[32] K. Mukai, R. Abe, B. J. Peterson, and S. Takayama. Improvement of infrared imaging video bolometer for application to deuterium experiment on the large helical device. Review of Scientific Instruments, 89(10):10E114, 2018.

[33] A. Sirinelli, F. Gandini, M. Henderson, J. W. Osterbeek, and G. Vayakis. Evaluation of EC stray radiation in ITER and its implications for diagnostics. Proceedings of Science, 240, 2016. DOI: https://doi.org/10.22323/1.240.0088.

[34] D. Zhang, R. Burhenn, R. König, L. Giannone, P. A. Grodzki, B. Klein, K. Grosser, J. Baldzuhn, K. Ewert, V. Erckman, M. Hirsch, H. P. Laqua, and J. W. Oosterbeek. Design criteria of the bolometer diagnostic for steady-state operation of the W7-X stellarator. Review of Scientific Instruments, 81:10E134, 2010.

[35] Y. M. Duan, S. T. Mao, L. Q. Hu, P. Xu, L. Q. Xu, J. Z. Zhang, and S. Y. Lin. Operation of bolometer system using Pt foil on $\mathrm{SiN}$ substrate detector for EAST tokamak. Review of Scientific Instruments, 87(11):11D434, 2016.

[36] S. Ullrich, H.-J. Hartfuss, M. Hirsch, and H. Laqua. 140-GHz test chamber for stray electron cyclotron radiation. Stellarator News, (98), May 2005.

[37] H. Meister, W. Kasparek, D. Zhang, M. Hirsch, J. Koll, and A. Zeitler. Millimetre wave attenuation of prototype diagnostic components for the ITER bolometers. Fusion Engineering and Design, 96-97:861 - 864, 2015. Proceedings of the 28th Symposium On Fusion Technology (SOFT-28).

[38] D. Zhang, H. Meister, L. Giannone, R. König, R. Burhenn, Th. Bergmann, D. Chauvin, P. Devynck, P.A. Grodzki, K. Höchel, Th. Sunn Pedersen. Thermal drift sudy on the bolometer diagnostic for steady-state fusion plasmas. Proceedings of the 39th EPS Conference, Stockholm 2012, Volume P5.040.

[39] F. Penzel, H. Meister, L. Giannone, and D. Zhang. Calibration parameter drift compensation of metal resistive bolometers operating in a thermal varying environment. Fusion Engineering and Design, 121:100 - 104, 2017.

[40] F. Penzel, H. Meister, N. Jaksic, H. Hermann, A. Pataki, L. C. Ingesson, and R. Reichle. Impact of thermal environment on metal resistor bolometers and concepts for compensation. to be submitted, 2019. Contribution to ECPD 2019.

[41] H. Meister, L. Giannone, L.D. Horton, G. Raupp, W. Zeidner, G. Grunda, S. Kalvin, U. Fischer, R. Reichle, A. Serikov, and S. Stickel. The ITER bolometer diagnostic - status and plans. Review of Scientific Instruments, 79:10F511-1 - 10F511-5, 2008.

[42] B. J. Peterson, R. Sano, M. L. Reinke, J. M. Canik, L. F. Delgado-Aparicio, J. D. Lore, K. Mukai, 
T. K. Gray, G. G. van Eden, and M. A. Jaworski. Preliminary design of a tangentially viewing imaging bolometer for NSTX-U. Review of Scientific Instruments, 87(11):11D410, 2016.

[43] W. Biel, M. de Baar, A. Dinklage, F. Felici, R. König, H. Meister, W. Treutterer, and R. Wenninger. Demo diagnostics and burn control. Fusion Engineering and Design, 96-97:8 - 15, 2015.

[44] W. Biel, R. Albanese, R. Ambrosino, M. Ariola, M.V. Berkel, I. Bolshakova, K.J. Brunner, R. Cavazzana, M. Cecconello, S. Conroy, A. Dinklage, I. Duran, R. Dux, T. Eade, S. Entler, G. Ericsson, E. Fable, D. Farina, L. Figini, C. Finotti, Th. Franke, L. Giacomelli, L. Giannone, W. Gonzalez, A. Hjalmarsson, M. Hron, F. Janky, A. Kallenbach, J. Kogoj, R. König, O. Kudlacek, R. Luis, A. Malaquias, O. Marchuk, G. Marchiori, M. Mattei, F. Maviglia, G. De Masi, D. Mazon, H. Meister, K. Meyer, D. Micheletti, S. Nowak, Ch. Piron, A. Pironti, N. Rispoli, V. Rohde, G. Sergienko, S. El Shawish, M. Siccinio, A. Silva, F. da Silva, C. Sozzi, M. Tardocchi, M. Tokar, W. Treutterer, and H. Zohm. Diagnostics for plasma control - from ITER to DEMO. Fusion Engineering and Design, 2019.

[45] W. Gonzalez, W. Biel, Ph. Mertens, M. Tokar, O. Marchuk, and Ch. Linsmeier. Conceptual studies on spectroscopy and radiation diagnostic systems for plasma control on DEMO. Fusion Engineering and Design, 2019. 